\title{
Fermionic topological quantum states as tensor networks
}

\author{
C. Wille, O. Buerschaper, and J. Eisert \\ Dahlem Center for Complex Quantum Systems, Freie Universität Berlin, 14195 Berlin, Germany
}

(Received 31 October 2016; revised manuscript received 3 April 2017; published 21 June 2017)

\begin{abstract}
Tensor network states, and in particular projected entangled pair states, play an important role in the description of strongly correlated quantum lattice systems. They do not only serve as variational states in numerical simulation methods, but also provide a framework for classifying phases of quantum matter and capture notions of topological order in a stringent and rigorous language. The rapid development in this field for spin models and bosonic systems has not yet been mirrored by an analogous development for fermionic models. In this work, we introduce a tensor network formalism capable of capturing notions of topological order for quantum systems with fermionic components. At the heart of the formalism are axioms of fermionic matrix-product operator injectivity, stable under concatenation. Building upon that, we formulate a Grassmann number tensor network ansatz for the ground state of fermionic twisted quantum double models. A specific focus is put on the paradigmatic example of the fermionic toric code. This work shows that the program of describing topologically ordered systems using tensor networks carries over to fermionic models.
\end{abstract}

DOI: 10.1103/PhysRevB.95.245127

\section{INTRODUCTION}

One of the longstanding questions of theoretical physics is concerned with the classification of phases of matter in quantum many-body systems [1]. In the recent past, the framework of tensor network states [2-6] has provided a particularly useful tool allowing a deepened understanding of quantum orders beyond Landau's symmetry-breaking paradigm. Notably, the phase classification of one-dimensional gapped systems has been essentially completed $[7,8]$ using tensor network states, in particular matrix-product states (MPS).

In two spatial dimensions and beyond, where the classification problem is much more intricate, tensor network states still provide a solid mathematical machinery. Indeed, beyond their use as variational states in powerful numerical methods, they constitute a concise framework for capturing notions of topological order. One reason for their usefulness is that a tensor network description admits easy access to the entanglement structure of a state. Entanglement theory is central to the understanding of topological order. Not surprisingly, in light of this observation, tensor network states give rise to a powerful tool in research located between condensed-matter physics and quantum information theory.

For spin and bosonic systems in two dimensions, an enormously fruitful approach to classifying quantum phases is based on a certain type of tensor network states, the projected entangled pair states (PEPS) [2-6,9-11]. A PEPS is a state vector,

$$
|\Psi\rangle=\sum_{i_{1}, \ldots, i_{N}} T\left[A^{i_{1}} \ldots A^{i_{N}}\right]\left|i_{1}, \ldots, i_{N}\right\rangle
$$

defined by tensors $A$ with a physical index $i_{k}$ and several virtual indices contracted according to a virtual lattice geometry (denoted by $T$ ). Each PEPS is the exact ground state of a respective parent Hamiltonian [12]. Hence, the emphasis is shifted from Hamiltonians to states, but the connection is kept with Hamiltonian problems by virtue of the parent. A natural question is to ask if and how phases of matter can be classified in terms of parent Hamiltonians of PEPS.
A first answer to that is given for so-called injective PEPS, which exhibit a one-to-one correspondence between the physical and virtual degrees of freedom [12]. This implies that they are unique ground states of their parent Hamiltonians and not yet able to incorporate topological order. After all, the most obvious topological invariant is a robust ground-state degeneracy which depends on the genus of the underlying surface.

However, it turns out that virtual symmetries of the tensor $A$ provide the key to an understanding of topological order. One can impose a group symmetry and require that the virtual and physical levels of any local tensor region are equivalent up to this group symmetry. This is known as $G$ injectivity [12] and leads to PEPS describing the topological order of discrete gauge theories or Kitaev's quantum double models [13], of which the famous toric code-the most paradigmatic model of intrinsic topological order and starting point for topological quantum memories-is an important example.

$G$ injectivity, however, is not the only mechanism which leads to topological order in tensor networks (and is not general enough to capture the models under consideration here). Indeed, the generalization of $G$ injectivity-matrixproduct operator (MPO) injectivity - constitutes a powerful framework for the understanding of topological order. A tensor is called MPO injective if it has a virtual symmetry given by a matrix-product projector $P$ such that the tensor viewed as a map from the physical to the virtual space can be inverted on the virtual symmetry subspace given by $P$ [cf. Fig. 1(b)]. MPO-injective PEPS [14-16] are able to represent larger classes of models, in particular they capture the famous Levin-Wen string-net models. This is remarkable progress: But all this applies to spin models (or bosonic ones, for that matter). Fermionic topological order remains unaddressed in this program, however, to date.

It is the purpose of this work to introduce a framework of tensor network states capable of capturing topological order in quantum lattice models having a fermionic component. In the focus of attention are tensor network states that exhibit fermionic MPO injectivity. We introduce axioms for matrixproduct injectivity that concisely capture the properties the 
(a)

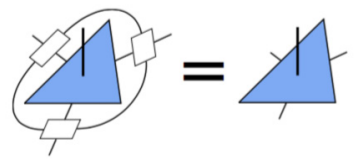

(b)

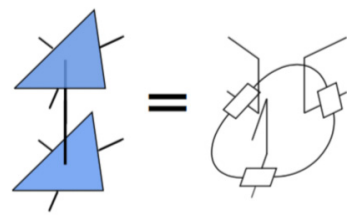

FIG. 1. (a) MPO symmetry, (b) MPO injectivity.

involved tensors must have, and hence provide a framework of tensor networks allowing one to describe fermionic topological order. We connect the general approach to a large class of physical models by discussing fermionic twisted quantum double models. Much in the focus of attention is the specific example of the paradigmatic fermionic toric code [17]. In that, we build upon insights that have been obtained on fermionic symmetry-protected topological order [18], as well as on frameworks to capture two-dimensional fermionic and bosonic topological order [8,19-22]. Notably, the latter body of work presents significant advances in the study of fermionic phases of matter, but does not make the connection to tensor networks yet, which allow for rigorous studies of properties of the underlying quantum states. This step is laid out in the present work.

\section{FERMIONIC TENSOR NETWORKS}

Tensor networks for purely fermionic systems have been considered without the connection to notions of topological order in the past [23-30], primarily motivated by numerical studies. Key to the framework developed here, however, is that the virtual symmetry of the tensors is incorporated in such a way that allows for studying notions of intrinsic fermionic topological order. It is this step that brings the study of phases of matter in terms of tensor networks to the fermionic realm that constitutes the main contribution of this work, identifying the required virtual symmetries. Tensor network descriptions are not even known for the paradigmatic fermionic toric code [17] that can be understood as the simplest fermionic string-net model [19] given as a two-dimensional lattice model with spins on the edges and fermions on the vertices. Reminiscent of the bosonic analog, its Hamiltonian $H=\sum_{v} Q_{v}+\sum_{p} Q_{p}$ is given by a sum of vertex operators $Q_{v}$ and plaquette operators $Q_{p}$ that are mutually commuting local projectors acting on bosonic and fermionic degrees of freedom simultaneously. The significant deviation from any bosonic model is reflected by the fact that the low-energy effective spin topological quantum field theory (TQFT) of the fermionic toric-code model is given by a Chern-Simons theory that cannot be realized by any local bosonic Hamiltonian. Even for this paradigmatic model, and more so in generality, significant obstacles to a tensor network description remain. It goes without saying that a naive embedding into a spin system is doomed to failure as a consequence of Jordan-Wigner strings. It is still much less clear to what extent ideas of MPO injectivity potentially carry over, given that the notion of locality is largely altered by the presence of fermionic anticommutation relations. That is to say, the introduction of fresh concepts of fermionic tensor networks are necessary, which will be developed from now on.

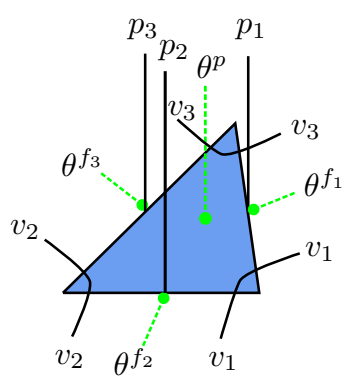

FIG. 2. Fermionic PEPS tensor $A$ given in Eq. (2). Solid black lines denote bosonic indices. Dashed green lines denote fermionic indices.

The key obstacle in constructing tensor networks for fermionic systems is to come up with a mechanism that allows the reordering of fermionic operators by keeping track of arising sign factors. Reordering the contraction order is a necessary prerequisite for the efficient contraction of a fermionic PEPS (fPEPS). One possibility to solve this problem is to use PEPS of increased bond dimension instead of fPEPS [31]. Another possibility is to use a particular kind of Grassmann number tensor network as, e.g., in the fermion coherent-state representation [32].

Here we use a similar construction. Each tensor has a physical fermionic mode $\theta^{p}$ and virtual fermionic modes $\theta^{f_{i}}$ represented by Grassmann numbers. In addition, the tensor has bosonic physical and virtual indices $p_{i}$ and $v_{i}$ and a bosonic weight which is used to perform sign-factor bookkeeping but also allows one to describe fermion-spin hybrid systems.

In the following, we will use tensors that are designed to describe fermion-spin hybrid models on trivalent graphs with spins on the edges and a fermionic mode at the vertex such as the fermionic twisted quantum double models addressed later. Different lattice geometries can be taken into account by blocking several tensors together. The tensors are of the form

$$
A=\sum A_{p f_{1} f_{2} f_{3}}^{p_{1} p_{2} p_{3} v_{2} v_{3}} \theta^{p} \theta^{f_{1}} \bar{\theta}^{f_{2}} \bar{\theta}^{f_{3}},
$$

as depicted in Fig. 2. When the sum of all Grassmann number exponents is even, the tensors commute and thus the tensor network state is independent of the contraction order.

A contraction along a Grassmann valued bond is defined by inserting a factor $\int d \bar{\theta}^{f} d \theta^{f}$ and integrating out the two Grassmann numbers $\theta^{f}$ and $\bar{\theta}^{f}$. To perform the integration, the Grassmann numbers have to be reordered, yielding sign factors that depend on the Grassmann numbers of the adjacent tensors. This has consequences for the virtual symmetries of the tensor, i.e., virtual symmetries cannot, in general, be captured by bosonic MPOs. This makes it necessary to introduce the concept of fermionic matrix-product operators.

\section{AXIOMS OF FERMIONIC MPO INJECTIVITY}

The key idea of fermionic MPO injectivity remains the same as in the bosonic setting: The MPO projector singles out a virtual subspace on every region, which eventually gives rise to the right entanglement scaling and the topological ground-state degeneracy. However, the algebraic structure of the fermionic symmetry MPO and the corresponding 

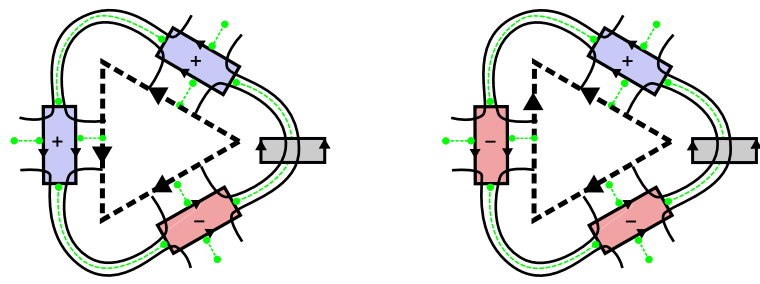

FIG. 3. Symmetry fMPOs $P_{+}$and $P_{-}$consisting of $Y$ tensors (gray) and tensors $T_{+}$(blue) and $T_{-}$(red).

virtual subspace is fundamentally different from the bosonic case. For example, performing a Jordan-Wigner transform to obtain a usual bosonic MPO would drastically alter the locality structure of the MPO. The virtual fermions also affect the stability of MPO symmetry and MPO injectivity under concatenation, since concatenation of two MPOs is always accompanied by a reordering of virtual modes and thus by emerging additional sign factors. In the following, we will define fermionic matrix-product operators and state the axioms of fermionic MPO injectivity.

Definition 1. fMPO symmetry. A tensor $A$ has fermionic MPO (fMPO) symmetry if it is invariant under the action of a fermionic MPO.

By fermionic MPO, we refer to an MPO consisting of single tensors that are fermionic, i.e., they have virtual bosonic bonds and virtual fermionic bonds, again represented by Grassmann numbers. To define MPO injectivity in more generality, it is convenient to equip the tensor network with a branching structure. That is, the edges of all PEPS tensors are oriented such that no cyclic triangles arise. The symmetry MPO, in general, depends on the orientation of the boundary edges of the tensor $A$ using the following construction: The MPO itself is given an orientation (counterclockwise used here) and consists of $T_{+}$tensors at edges that are parallel to the MPO direction and $T_{-}$tensors at antiparallel edges. Using two types of tensors to construct general MPOs suffices in the bosonic setting [14]. However, in the fermionic setting, additional sign factors emerging from a reordering of fermionic modes pose an obstacle to the stability of MPO symmetry under concatenation - an axiom that is required. In order to overcome this adversity and consistently define symmetry MPOs for a region $R$ composed of an arbitrary number of MPOsymmetric triangle tensors, one can introduce an additional purely bosonic sign-factor tensor $Y$ whose position within the MPO depends on the edge orientations on the boundary of $R$. To illustrate the construction, the symmetry MPOs for the two possible branching structures of a single triangle tensor $P_{+}=\mathrm{t} \operatorname{Tr}\left[T_{+} T_{+} T_{-} Y\right]$ and $P_{-}=\operatorname{tTr}\left[T_{+} T_{-} T_{-} Y\right]$ are depicted in Fig. 3. Here, $\mathrm{t} T r$ denotes a contraction of all bonds along the transversal direction of the MPO ring.

Axiom 1. Projector property. The MPOs $P_{ \pm}$fulfill $P_{ \pm}^{2}=P_{ \pm}$ and thus are projectors.

Based on the stability of MPO injectivity under concatenation explained below, this property generalizes to MPOs of arbitrary size.

Axiom 2. Stability of $f M P O$ symmetry. Fermionic MPO symmetry is stable under concatenation.

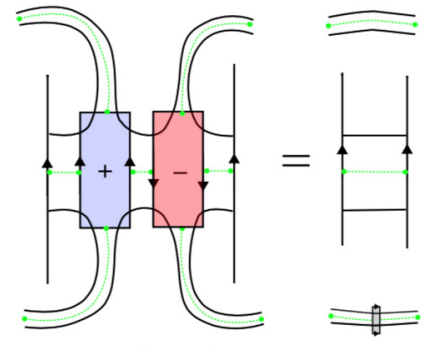

(a) Open boundary

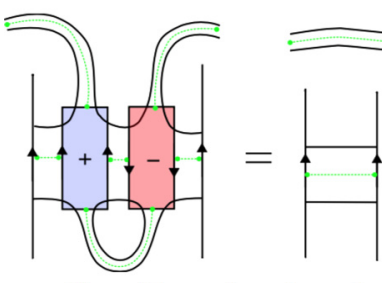

(b) Closed boundary type I

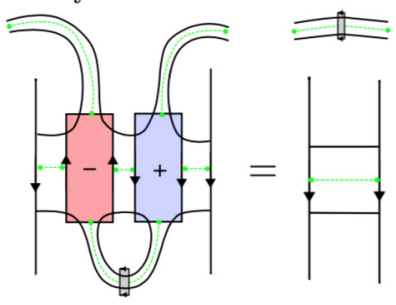

(c) Closed boundary type II
FIG. 4. Concatenation of two MPO tensors with open or partially closed radial indices.

In particular, the concatenation of two fMPO-symmetric tensors with compatible branching structure is again fMPO symmetric. In order for this axiom to be realizable, one first needs to consistently define symmetry fMPOs on regions of arbitrary size. One way to do this is to choose a branching structure that admits a global flow direction, i.e., all edges are oriented into one direction with a deviation of less than $\pi / 2$, and to place $Y$ tensors within the MPO at positions that depend on the edge orientation of the boundary. Then one can show that if the concatenation of two MPO tensors proceeds according to the rules stated in Fig. 4, stability under concatenation is guaranteed. For more details and a proof based on induction, we refer to the Appendix.

Definition 2. $F M P O$ injectivity. A tensor $A$ that is fMPO symmetric and has a pseudoinverse $\tilde{A}$ such that $\tilde{A} A=P$, i.e., is invertible on the fMPO-symmetric subspace given by the projector $P$, is called $f M P O$ injective.

Axiom 3. Stability of fMPO injectivity. Fermionic MPO injectivity is stable under concatenation, i.e., the concatenation of two fMPO-injective tensors is again fMPO injective.

As in the bosonic case, this is a direct consequence of the stability of fMPO symmetry under concatenation [14].

Tensor networks satisfying fermionic MPO injectivity defined by the three axioms above provide a consistent and versatile framework to describe nonchiral intrinsic topological order for fermionic models. To provide further substance to the framework established, we present a large class of fermionic models that admit an fMPO-injective tensor network description.

\section{FERMIONIC TWISTED QUANTUM DOUBLE MODELS}

In the realm of bosonic MPO injectivity, it has been established that the ground states of an important class of models exhibiting topological order, i.e., Levin-Wen string-net models, can be written as PEPS. Furthermore, the selfconsistency equations of the renormalization-group (RG) flow defined for Levin-Wen string-net models, in particular the 
pentagon equation, give rise to a virtual MPO symmetry of the respective tensors.

Building upon this insight, we construct fPEPS for a particular subset of fermionic string-net models proposed in Ref. [19] that we refer to as fermionic twisted quantum double models. Here, the bosonic spin degrees of freedom (edge labels) are given by a group $G$ and spinless fermions on the vertices are coupled to the bosonic edge degrees via a 2-cocycle $s \in \mathcal{H}^{2}\left(G, \mathbb{Z}_{2}\right)$ fulfilling

$$
\operatorname{Mod}[s(a, b)+s(a b, c)+s(a, b c)+s(b, c), 2]=0 .
$$

That is, the presence of a fermion at a vertex is determined by the adjacent spins on the two incoming edges or the two outgoing edges $g_{i}, g_{j}$ of the vertex via $\theta^{s\left(g_{i}, g_{j}\right)}$.

For bosonic twisted quantum double models, the main self-consistency equation of the RG flow, i.e., the pentagon equation, reduces to a 3-cocycle equation. In the fermionic setting, the anticommutation relations lead to a super-3cocycle equation, i.e., a 3-cocycle equation graded by the 2-cocycle $s$,

$$
\begin{aligned}
& \omega(a, b, c) \omega(a, b c, d) \omega(b, c, d) \\
& \quad=(-1)^{s(a, b) s(c, d)} \omega(a b, c, d) \omega(a, b, c d) .
\end{aligned}
$$

A solution to Eq. (4) exists iff the function $(-1)^{s(a, b) s(c, d)}$ is a coboundary $\mathcal{B}^{4}(G, U(1))$ viewed as a 4 -cocycle. The full model is then described in terms of a triple $(G, s, \omega)$, where $\omega \in \mathcal{H}_{f}^{3}(G, U(1), s)$ fulfills Eq. (4).

We use the branching structure of the lattice to define positively and negatively oriented tensors $A_{+/-}$according to whether the majority of the edges are oriented clockwise or counterclockwise (cf. Fig. 3 ). The expressions for $A_{ \pm}$read

$$
\begin{aligned}
A_{+}= & \sum_{v_{0}, v_{1}, v_{2}} \omega\left(v_{0}, v_{0}^{-1} v_{1}, v_{1}^{-1} v_{2}\right) \\
& \times \theta^{s\left(v_{0}^{-1} v_{1}, v_{1}^{-1} v_{2}\right)} \theta^{s\left(v_{0}, v_{0}^{-1} v_{2}\right)} \bar{\theta}^{s\left(v_{1}, v_{1}^{-1} v_{2}\right)} \bar{\theta}^{s\left(v_{0}, v_{0}^{-1} v_{1}\right)} \\
& \times\left|v_{0}^{-1} v_{1}, v_{1}^{-1} v_{2}, v_{0}^{-1} v_{2}\right\rangle\left\langle v_{0}, v_{1}, v_{2}\right|, \\
A_{-}= & \sum_{v_{0}, v_{1}, v_{2}} \omega^{-1}\left(v_{0}, v_{0}^{-1} v_{1}, v_{1}^{-1} v_{2}\right) \\
& \times \theta^{s\left(v_{0}, v_{0}^{-1} v_{1}\right)} \theta^{s\left(v_{1}, v_{1}^{-1} v_{2}\right)} \bar{\theta}^{s\left(v_{0}, v_{0}^{-1} v_{2}\right)} \bar{\theta}^{s\left(v_{0}^{-1} v_{1}, v_{1}^{-1} v_{2}\right)} \\
& \times\left|v_{0}^{-1} v_{1}, v_{1}^{-1} v_{2}, v_{0}^{-1} v_{2}\right\rangle\left\langle v_{0}, v_{1}, v_{2}\right| .
\end{aligned}
$$

The 2-cocycle $s$ determines the occupation of physical (virtual) fermionic modes via a coupling to their adjacent physical (virtual) spins which ensures that the fPEPS tensors are commuting by construction. Reminiscent of the bosonic setting, the weight of the tensors is defined in terms of the super-3-cocycle $\omega$ and the relation between physical and virtual indices is chosen as $p_{i, j}=v_{i}^{-1} v_{j}$, which means that the fPEPS is not injective, but invertible only on a symmetry subspace singled out by the MPO projector given below.

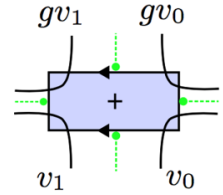

(a)

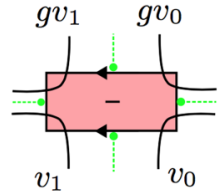

(b)

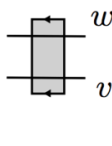

(c)
FIG. 5. The MPO tensors $T_{+}(g), T_{-}(g)$, and $Y$.

Next we explicitly construct the $T_{+}$and $T_{-}$tensors of the fMPO under which $A_{ \pm}$is symmetric [cf. Figs. 5(a) and 5(b)],

$$
\begin{aligned}
T_{+}(g)= & \sum_{v_{0}, v_{1}} \omega\left(g, v_{0}, v_{0}^{-1} v_{1}\right) \\
& \times \theta^{s\left(v_{0}, v_{0}^{-1} v_{1}\right)} \theta^{s\left(g, v_{1}\right)} \bar{\theta}^{s\left(g v_{0}, v_{0}^{-1} v_{1}\right)} \bar{\theta}^{s\left(g, v_{0}\right)}, \\
T_{-}(g)= & \sum_{v_{0}, v_{1}} \omega^{-1}\left(g, v_{1}, v_{1}^{-1} v_{0}\right) \quad\left|v_{0}, v_{1}\right\rangle\left\langle g v_{1}\right| \\
& \times \theta^{s\left(g, v_{1}\right)} \theta^{s\left(g v_{1}, v_{1}^{-1} v_{0}\right)} \bar{\theta}^{s\left(g, v_{0}\right)} \bar{\theta}^{s\left(v_{1}, v_{1}^{-1} v_{0}\right)},
\end{aligned}
$$

and the purely bosonic sign-factor tensor depicted in Fig. 5(c),

$$
Y=\sum_{v, w}(-1)^{s\left(w v^{-1}, v\right)}|v, w\rangle\langle v, w| .
$$

Note that each group element $g \in G$ yields a distinct symmetry MPO, i.e., we have $A_{ \pm}=A_{ \pm} V_{ \pm}(g)$, with $V_{ \pm}(g)=$ $\mathrm{t} \operatorname{Tr}\left[T_{ \pm}(g) T_{ \pm}(g) T_{\mp}(g) Y\right]$ for each element independently. The MPO projector is given by $P_{ \pm}=\sum_{g} V_{ \pm}(g)$ and the projector identity $P_{ \pm}^{2}=P_{ \pm}$follows from the fact that $V_{ \pm}(g)$ fulfills the group representation property $V_{ \pm}(g) V_{ \pm}(h)=V_{ \pm}(h g)$. The existence of the pseudoinverses of $A_{ \pm}$is verified by explicit calculation and the stability of MPO symmetry and MPO injectivity under concatenation follows directly from the fact that the tensors $T_{ \pm}$fulfill the concatenation properties depicted in Fig. 4, which is a consequence of direct calculation.

\section{GROUND-STATE SPACE}

The concept of fMPO injectivity makes it possible to compute essential properties of a physical model solely based on the characterization of the virtual tensor symmetries without considering the state or the Hamiltonian explicitly on a physical level. Based on the formalism developed, one can compute the ground-state degeneracy of an fMPO-injective PEPS on a torus equipped with a branching structure admitting a global flow, without having to consider the physical degrees of freedom. Here, we closely follow the approach of Ref. [14]. We consider all locally undetectable closures on a minimal torus which are MPO symmetric, i.e., all states which can be defined simultaneously using the three different closure tensors depicted in Fig. 6. Similar to the bosonic setting, one can parametrize this space as

$$
\operatorname{span}\{M(g, h) \mid[g, h]=0, s(g, h)=s(h, g)\},
$$



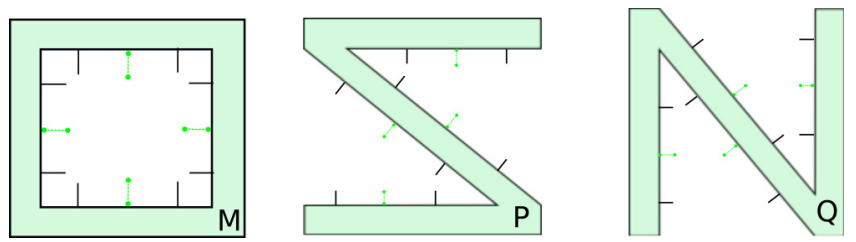

FIG. 6. The three different closure tensors on a minimal torus.

where the closure tensor $M$ is constructed as depicted in Fig. 7 and given by

$$
\begin{aligned}
M(g, h)= & \sum_{\alpha} \lambda(\alpha ; g, h)|\alpha, g \alpha, g h \alpha, h \alpha\rangle \\
& \times \theta^{s\left(g \alpha,{ }^{\alpha} g^{-1}\right)} \theta^{s\left(h g \alpha,{ }^{\alpha} h^{-1}\right)} \bar{\theta}^{s\left(g h \alpha,{ }^{\alpha} g^{-1}\right)} \bar{\theta}^{s\left(h \alpha,{ }^{\alpha} h^{-1}\right)} .
\end{aligned}
$$

Here, ${ }^{\alpha} g=\alpha^{-1} g \alpha$ denotes conjugation by the inverse element and the coefficient $\lambda$ is given in the Appendix.

Imposing MPO symmetry simply by acting with a four-site MPO $V(k)$ on $M$, we obtain a parametrization of the groundstate space in terms of MPO-symmetric tensors,

$$
\begin{aligned}
M^{\prime}(g, h) & =\frac{1}{|G|} \sum_{k} V(k) M(g, h) \\
& =\frac{1}{|G|} \sum_{k} \eta_{g}^{\omega}(h, k) M\left(g^{k}, h^{k}\right),
\end{aligned}
$$

where $g^{k}=k g k^{-1}$ denotes conjugation and $\eta_{g}^{\omega}(h, k)$ is given in the Appendix.

Next we count the number of linearly independent elements of the ground-state space. First, one can show that two states $M^{\prime}(g, h)$ and $M^{\prime}(k, l)$ are linearly dependent if $(g, h)$ and $(k, l)$ are in the same pair-conjugacy class. Furthermore, not all pair-conjugacy classes correspond to a nonvanishing state. Similar to the bosonic setting, one finds that only pairconjugacy classes $\mathcal{C}(g, h)$ contribute to the total ground-state space dimension for which $[g, h]=0, s(g, h)=s(h, g)$ and, for all elements of the centralizer $k \in \mathcal{Z}(g, h)$, we have

$$
c_{g}^{\omega}(h, k)=c_{g}^{\omega}(k, h),
$$

where

$$
c_{g}^{\omega}(h, k)=\frac{\omega(g, h, k) \omega(h, k, g)}{\omega(h, g, k)} .
$$

We refer to this property as $\left(c^{\omega}, s\right)$ regular. Since states from different pair-conjugacy classes are linearly independent,
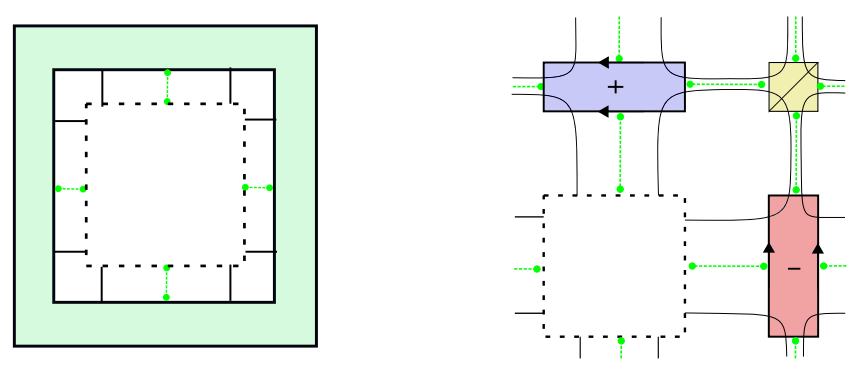

FIG. 7. Closure tensor $M(g, h)$. we conclude that the ground-state degeneracy for fermionic twisted quantum double models defined by a triple $(G, s, \omega)$ on a minimal torus is given by the number of $\left(c^{\omega}, s\right)$-regular pair-conjugacy classes. In complete analogy to the bosonic setting, the considerations on the minimal torus hold equally well on a torus of arbitrary size due to the axioms of stability under concatenation.

In the case where the 2-cocycle $s$ is trivial, i.e., the bosonic case, we obtain a classification of the ground-state basis in terms of $c^{\omega}$-regular pair-conjugacy classes as expected from the results of Ref. [14] for bosonic twisted quantum double models. We recall that the ground-state degeneracy of a bosonic quantum double model for a group $G$ is given by the number of pair-conjugacy classes and that the ground-state degeneracy for any twisted bosonic quantum double model is thus always lesser than or equal to the degeneracy of the untwisted model. For fermionic twisted quantum double models, there is no untwisted counterpart because for a fermionic model there is at least one pair of group elements $(a, b)$ for which the 2-cocycle evaluates to $s(a, b)=1$ in order to be called a fermionic model. This implies that the graded 3 -cocycle $\omega$ cannot be chosen to be completely trivial. Thus, we cannot compare the ground-state degeneracy of twisted fermionic quantum double to untwisted fermionic quantum double models. However, it remains true that the groundstate degeneracy of a twisted fermionic quantum double model will be lesser than or equal to the untwisted bosonic quantum double. Also note that not all bosonic quantum double models have fermionic counterparts, i.e., for $\mathbb{Z}_{n}$ with $n$ odd, there is no nontrivial 2-cocycle, i.e., no fermionic model. It is an open question whether the symmetry condition for the 2-cocycle $s(g, h)=s(h, g)$ is a substantial restriction on the pair-conjugacy classes on top of the $c^{\omega}$ regularity. A simple calculation shows that for cyclic groups, all 2-cocycles are symmetric and the condition is trivially fulfilled, but it remains an interesting open question whether this result extends to all Abelian groups.

\section{FERMIONIC TORIC CODE}

To illustrate the formalism of fermionic MPO-injective fPEPS, we will elucidate how the ground state of the fermionic toric-code Hamiltonian as proposed in Ref. [17] can be written as a fermionic tensor network satisfying the axioms of fermionic MPO injectivity. The fermionic toric-code Hamiltonian is given in a string-net description. It can be seen as the simplest solution of the self-consistency equations for fermionic twisted quantum double models, i.e., the simplest triple $(G, s, \omega)$.

The local Hilbert space is given by qubits represented by the group $\mathbb{Z}_{2}=\{0,1\}$. This group has only two second cohomology classes. Choosing the 2-cocycle $s$ as a representative of the trivial cohomology class, one obtains the usual bosonic toric-code or the double-semion model, depending on the choice of $\omega$, i.e., the solution to the pentagon equation which is a usual 3-cocycle equation in this case. Choosing the nontrivial second cohomology class, in particular the normalized representative $s_{f T C}(g, h)=1$ if $g=h=1$ and 0 otherwise, yields two normalized solutions to the graded 
pentagon equation,

$$
\omega_{f T C}(g, h, k)= \begin{cases} \pm i & \text { if } g=h=k=1 \\ 1 & \text { otherwise, }\end{cases}
$$

which precisely correspond to the fermionic toric-code model and its dual. For a discussion of possible Hamiltonian gauges for the fermionic toric code, see the Appendix.

The ground-state wave function of the fermionic toric code can be written in terms of $A_{ \pm}$tensors, defined in Eq. (5) and (6) using $s$ and $\omega$, and the lattice geometry dual to the physical lattice from Ref. [17] which admits a global flow. By construction, this tensor network is fMPO injective. To prove that it is indeed the ground state of the fermionic toric-code Hamiltonian, one can verify that the tensor network is an eigenstate to each local projector. The property of being an eigenstate to the vertex projector is also already implied in the construction of the $A_{ \pm}$tensor. Confirmation that the tensor network state is an eigenstate to each plaquette operator is done by explicit calculation in the Appendix. Intuitively, this can be understood by interpreting the plaquette operator as inserting a closed loop around the center of the hexagon. The contraction of the inner virtual index $v_{0}$ exactly compensates for that, i.e., $Q_{p} A_{\text {hex }}\left(v_{0}=0\right)=A_{\text {hex }}\left(v_{0}=1\right)$ and $Q_{p} A_{\text {hex }}\left(v_{0}=1\right)=A_{\text {hex }}\left(v_{0}=0\right)$. Note that due to the anticommutating Grassmann variables, it is not trivial to see that all sign factors are indeed correct, but it is a result of an explicit calculation. Due to the translation invariance of the lattice, it follows that the tensor network state is the ground state on any region with open virtual boundary. To calculate the ground-state degeneracy on a torus, we use the formalism developed in the previous section. Since $\mathbb{Z}_{2}$ is Abelian, there are four pair-conjugacy classes labeled by $(0,0),(0,1),(1,0)$, and $(1,1)$. All of them are $\left(c^{\omega}, s\right)$ regular. To see this, note that the 2-cocycle $s$ is symmetric, which is true for any cyclic group, as noted earlier. The graded 3-cocycle is normalized. Thus, the regularity condition is trivially fulfilled whenever one of the elements $g, h, k$ is the trivial group element, but the only other case $g=h=k=1$ is also trivially fulfilled. Thus, the ground-state degeneracy on a torus is four, which is in agreement with the result obtained directly on the physical level in Ref. [17].

\section{SUMMARY AND OUTLOOK}

In this work, we have introduced a general tensor network formalism that is sufficiently versatile to capture topological order of quantum systems with a fermionic component. We hence generalize the idea of describing phases of matter using tensor network states to the fermionic realm. Yet, needless to say, this is only the beginning of an extensive program: In future work, instances of symmetry-protected topological order will be discussed, as well as more subtle situations in which a global flow cannot be identified [33]. It will also be interesting to grasp modular matrices directly in this framework. It is the hope that this work can be seen as a further invitation to explore tensor networks to capture topological phases of matter.

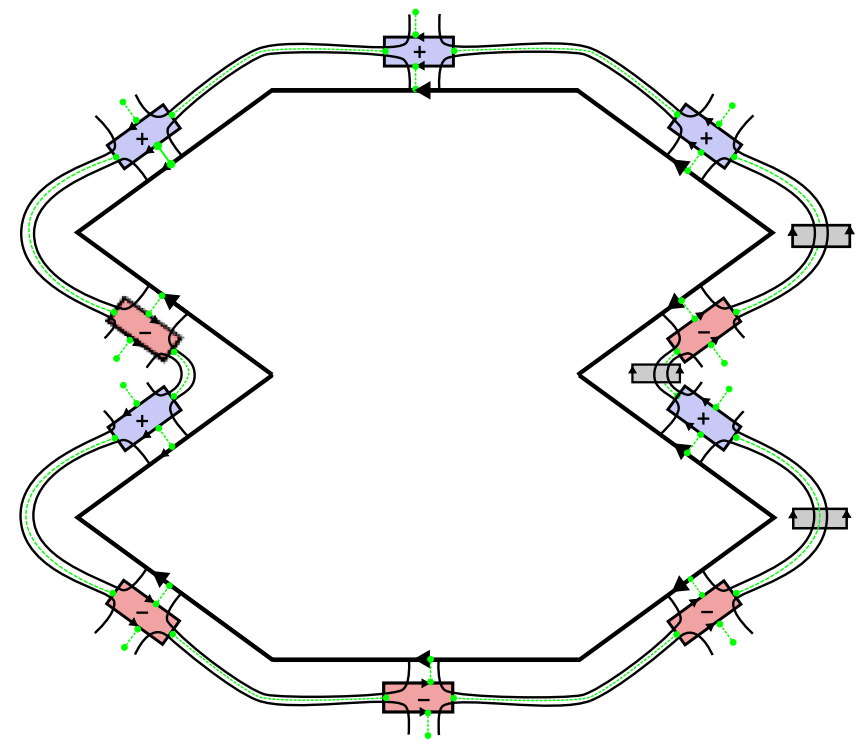

FIG. 8. MPO for a generic boundary consisting of $Y$ tensors (gray) and tensors $T_{+}$(blue) and $T_{-}$(red).

\section{ACKNOWLEDGMENTS}

We would like to thank the DFG (Grant No. CRC 183), the Templeton Foundation, the Studienstiftung des Deutschen Volkes, and the ERC (Grant No. 307498) (TAQ) for support.

\section{APPENDIX A: CONCATENATION STABILITY}

We restate the construction recipe for consistent MPOs given a branching structure admitting a global flow: $Y$ tensors are placed at each point where the boundary vertex has two outgoing edges, which form an angle of less than $\pi$, and at each point where the boundary vertex has two incoming edges, which form an angle of more than $\pi$. In Fig. 8, a tensor with a generic boundary and its symmetry MPO are depicted to illustrate the construction. Next we provide proof that the stability of MPO symmetry under concatenation is guaranteed if the concatenation of two MPOs follows the rules stated in Fig. 4. This is done by induction and it suffices to show that adding MPO-symmetric triangle tensors to an existing MPO-symmetric tensor patch is again MPO symmetric. This is easily checked by considering all different concatenation cases. To this end, we first make a distinction between an "open" concatenation, where the MPO tensor concatenation depicted in Fig. 4(a) is relevant, and a partially closed concatenation [Figs. 4(b) and 4(c)], relevant when an outer vertex of a triangle tensor becomes an inner vertex during the concatenation process.

Before we discuss the different cases occurring, we introduce a shorthand notation to symbolize the concatenation of MPO-symmetric tensors. Instead of drawing the full symmetry MPO, we just indicate the positions of $Y$ tensors before and after the concatenation (Fig. 9) by circles at the respective boundary vertices.

In the case of "open" concatenation, it is sufficient to consider concatenation along an edge pointing towards the global flow direction and then categorize the different cases according to the shared angles with the neighboring edges 

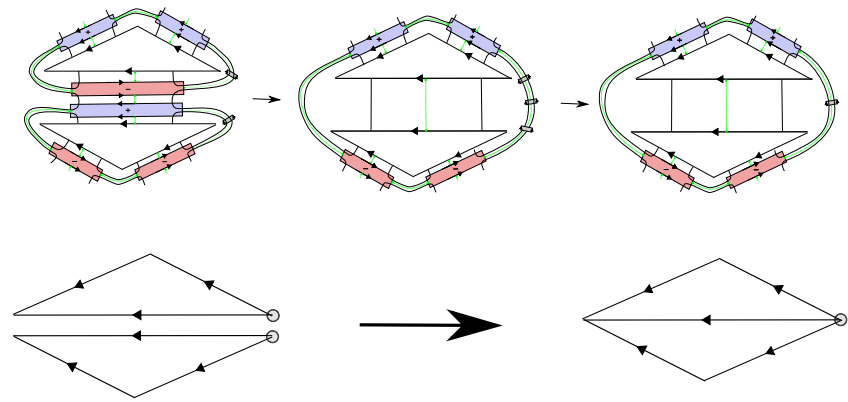

FIG. 9. The concatenation of two MPO-symmetric tensors written in explicit notation (upper panel) and in abbreviated notation only depicting the positions of $Y$ tensors.

at the origin vertex. Since the placement of $Y$ tensors only depends on the criteria of whether angles are smaller or larger than $\pi$ and the edge orientations, the exact angles are irrelevant and we only have to distinguish four cases distinguished by $0, \pi / 2, \pi, 3 \pi / 2$. Taking into account geometric constraints imposed by the global flow criterion and making use of the mirror symmetry along the global flow direction, there are six distinct cases left to consider. All of them can be shown to yield the correct MPO symmetry after concatenation, as shown in Fig. 10.

The second case is relevant when two MPO-symmetric tensors are contracted along a common boundary of length two or more. Performing the contraction sequentially along the common boundary amounts to a step-by-step reduction of the size of the boundary and thus to a reduction of the size of the symmetry MPO. Note that in this case, there are two different concatenation rules depending on the orientation of the inner edge relative to the position of the already contracted transversal indices [Figs. 4(b) and 4(c)].

If the edges to be contracted share a vertex at their origin, the contraction does not yield additional $Y$ tensors and, independently from the rest of the tensor, the contracted tensor will have the expected MPO symmetry (Fig. 11, upper panel). In the other case, one can consider all different cases of the edge configurations in the immediate vicinity of the contracted edge. Using symmetry arguments, there are three distinct cases which can be checked explicitly (Fig. 11, lower three panels).

\section{APPENDIX B: GROUND-STATE SPACE}

In this section, we present additional details to the calculation of the ground-state space degeneracy. The coefficient $\lambda$ in Eq. (11) is given by

$$
\begin{aligned}
\lambda(\alpha ; g, h)= & \frac{\omega(h, g, \alpha) \omega\left(h, g \alpha,{ }^{\alpha} g^{-1}\right)}{\omega(g, h, \alpha) \omega\left(g, h \alpha,{ }^{\alpha} h^{-1}\right)} \\
& \times(-1)^{s\left(g h \alpha,{ }^{\alpha} h^{-1}\right) s\left(g \alpha,{ }^{\alpha} g^{-1}\right)+s\left(h g \alpha,{ }^{\alpha} g^{-1}\right) s\left(h \alpha,{ }^{\alpha} h^{-1}\right)} \\
& \times(-1)^{\left(s(h, \alpha)+s\left(h \alpha,{ }^{\alpha} h^{-1}\right)\right)\left(s(g, \alpha)+s\left(g \alpha,{ }^{\alpha} g^{-1}\right)\right)+s(h g, \alpha)},
\end{aligned}
$$
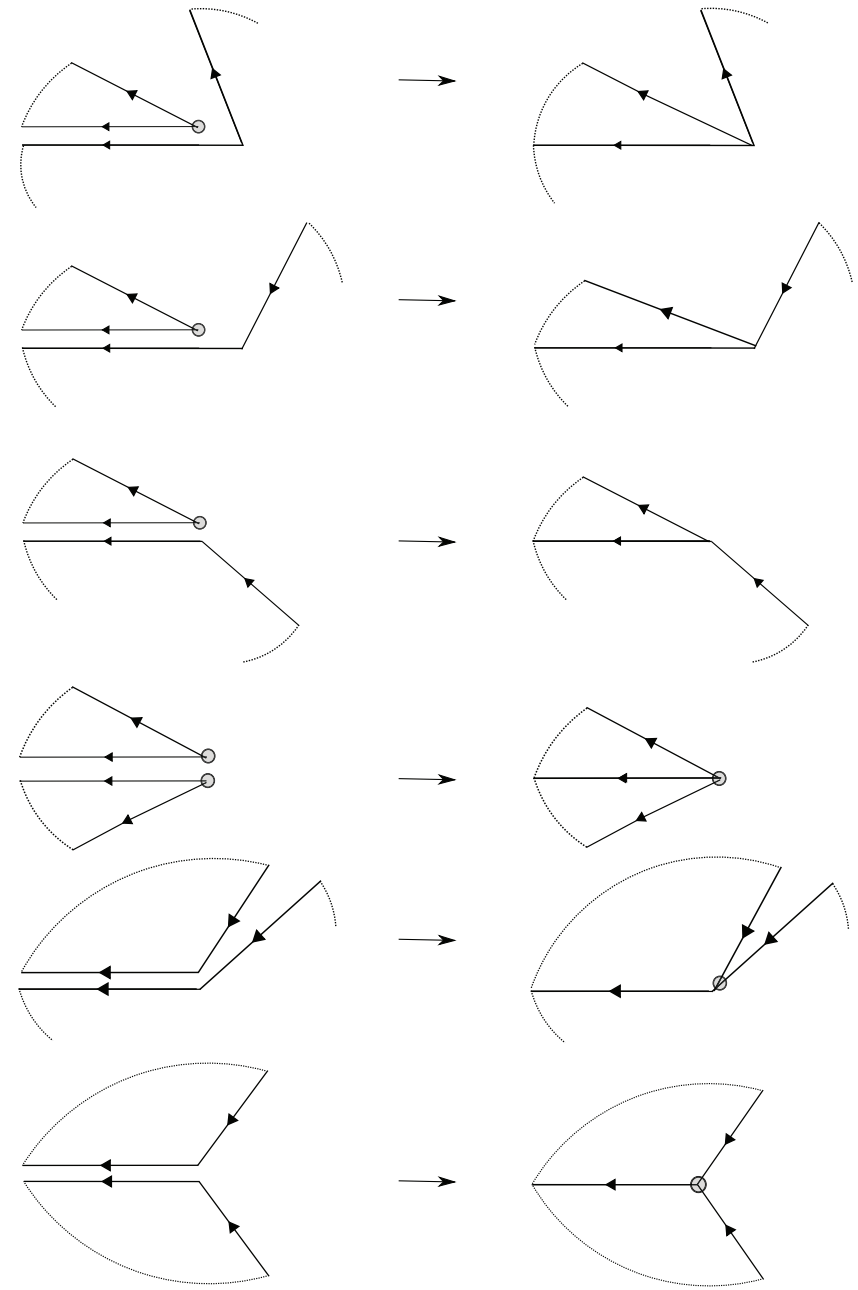

FIG. 10. All six distinct cases for concatenating two MPOsymmetric tensors along an open edge.

and the coefficient $\eta_{g}^{\omega}(h, k)$ in Eq. (12) by

$$
\begin{aligned}
\eta_{g}^{\omega}(h, k)= & \frac{\omega\left(g, k^{-1}, h^{k}\right) \omega\left(k^{-1}, h^{k}, g^{k}\right) \omega\left(h, g, k^{-1}\right)}{\omega\left(h, k^{-1}, g^{k}\right) \omega\left(k^{-1}, g^{k}, h^{k}\right) \omega\left(g, h, k^{-1}\right)} \\
& \times(-1)^{\left[s\left(k^{-1}, k h\right)+s\left(k h, k^{-1}\right)\right]\left[s\left(k^{-1}, k g\right)+s\left(k g, k^{-1}\right)\right]} \\
& \times(-1)^{s\left(k^{-1}, k g h\right)+s\left(k g h, k^{-1}\right)} .
\end{aligned}
$$

The fact that $M^{\prime}(g, h)$ and $M^{\prime}(j, l)$ are linear dependent if $(j, l)=\left(g^{t}, h^{t}\right)$ for some $t$, i.e., if they are in the same pairconjugacy class, follows from the identity

$$
\eta_{g^{t}}^{\omega}\left(x^{t}, y t^{-1}\right)=\frac{\eta_{g}^{\omega}(x, y)}{\eta_{g}^{\omega}(x, t)}
$$

that holds formally as in the bosonic setting despite the fact that $\eta^{\omega}$ has additional sign factors and is given by a product of super 3 -cocycles $\omega$. Equation (B3) is also used in order to derive that only $\left(c^{\omega}, s\right)$-regular pair-conjugacy classes contribute to the ground-state dimension. To this end, first note that if

$$
\sum_{s \in Z(g, h)} \eta_{g}^{\omega}(h, s)=0,
$$




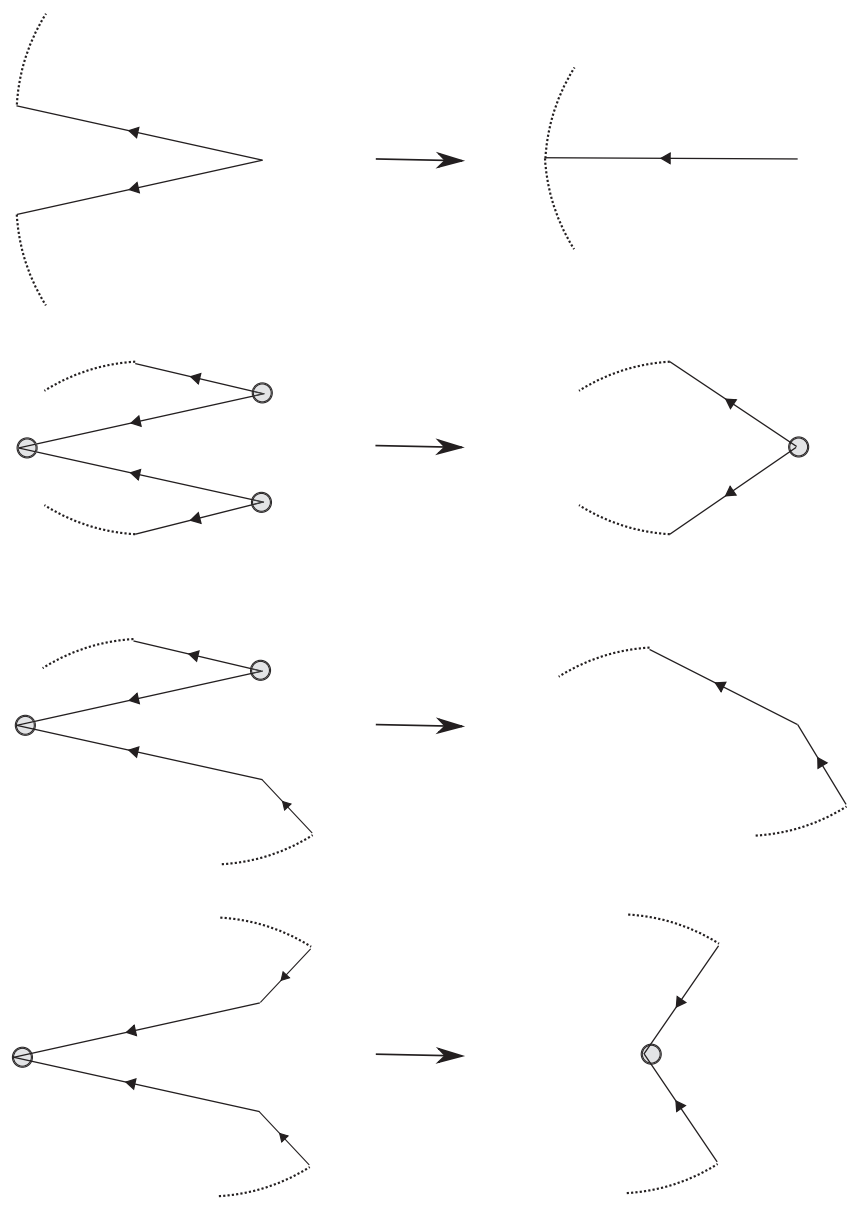

FIG. 11. Contracting two edges of the same MPO-symmetric tensor. then also

$$
\sum_{s \mid g^{s}=g^{t_{i}}, h^{s}=h^{t_{i}}} \eta_{g}^{\omega}(h, s)=0, \quad \forall i .
$$

Writing out the state $M^{\prime}(g, h)$ in the basis of the elements of the pair-conjugacy class $\mathcal{C}(g, h)$, we make use of the fact above to conclude that

$$
M^{\prime}(g, h)=0 \Leftrightarrow \sum_{s \in \mathcal{Z}(g, h)} \eta_{g}^{\omega}(h, s)=0 .
$$

To single out the pair-conjugacy classes for which Eq. (B6) is fulfilled, we note that for all elements $k$ in the centralizer of $(g, h)$ with $[g, h]=0$, we have

$$
\eta_{g}^{\omega}(h, k)=\frac{c_{g}^{\omega}\left(k^{-1}, h\right)}{c_{g}^{\omega}\left(h, k^{-1}\right)},
$$

where $c_{g}^{\omega}(h, k)$ as defined in Eq. (14). For $g, h, k \in \mathcal{Z}(g, h)$ and $[g, h]=0$, i.e., $g, h, k$ are mutually commuting, $c_{g}^{\omega}(h, k)$ is a 2 -cocycle. This insight is used to apply the same arguments as in Ref. [34] and show that

$$
\sum_{s \in \mathcal{Z}(g, h)} \eta_{g}^{\omega}(h, s)=0 \Leftrightarrow c_{g}^{\omega}(h, s) \neq c_{g}^{\omega}(s, h) .
$$

In other words, only pair-conjugacy classes $\mathcal{C}(g, h)$ contribute to the total ground-state space dimension for which $[g, h]=$ $0, s(g, h)=s(h, g)$ and $c_{g}^{\omega}(h, k)=c_{g}^{\omega}(k, h)$.

\section{APPENDIX C: HAMILTONIAN GAUGE}

As addressed in Ref. [20], string-net models can be defined using different gauges. Under such gauge transformations, the Hamiltonian changes according to local physical unitary operations that do not alter the topological phase. A typical gauge degree of freedom is the choice of the loop weight, also

\begin{tabular}{|c|c|c|c|}
\hline$i, j, k, l, m, n$ & $p(i, j, k, l, m, n) \mathcal{F}_{p}(i, j, k, l, m, n)$ & $i, j, k, l, m, n$ & $p(i, j, k, l, m, n) \mathcal{F}_{p}(i, j, k, l, m, n)$ \\
\hline 000000 & $-\alpha / \beta c_{3}^{\dagger} c_{6}^{\dagger}$ & & \\
\hline 100000 & $c_{3}^{\dagger} c_{1}$ & 010000 & $1 / \beta^{2} c_{1}^{\dagger} c_{2}^{\dagger} c_{3}^{\dagger} c_{6}^{\dagger}$ \\
\hline 001000 & $c_{6}^{\dagger} c_{2}$ & 000100 & $c_{6}^{\dagger} c_{4}$ \\
\hline 000010 & $-1 / \beta^{2} c_{3}^{\dagger} c_{4}^{\dagger} c_{5}^{\dagger} c_{6}^{\dagger}$ & 000001 & $c_{3}^{\dagger} c_{5}$ \\
\hline 110000 & $-1 / \beta c_{2}^{\dagger} c_{3}^{\dagger}$ & 011000 & $-1 / \beta c_{1}^{\dagger} c_{6}^{\dagger}$ \\
\hline 001100 & $-\beta c_{6}^{\dagger} c_{4} c_{3} c_{2}$ & 000110 & $1 / \beta c_{5}^{\dagger} c_{6}^{\dagger}$ \\
\hline 000011 & $-1 / \beta c_{3}^{\dagger} c_{4}^{\dagger}$ & 100001 & $\beta c_{3}^{\dagger} c_{6} c_{5} c_{1}$ \\
\hline 101000 & $-\alpha \beta c_{2} c_{1}$ & 010100 & $\alpha / \beta c_{1}^{\dagger} c_{2}^{\dagger} c_{6}^{\dagger} c_{4}$ \\
\hline 001010 & $-\alpha / \beta c_{4}^{\dagger} c_{5}^{\dagger} c_{6}^{\dagger} c_{2}$ & 000101 & $\alpha \beta c_{5} c_{4}$ \\
\hline 100010 & $-\alpha / \beta c_{3}^{\dagger} c_{4}^{\dagger} c_{5}^{\dagger} c_{1}$ & 010001 & $\alpha / \beta c_{1}^{\dagger} c_{2}^{\dagger} c_{3}^{\dagger} c_{5}$ \\
\hline 100100 & $-\alpha \beta c_{4} c_{1}$ & 010010 & $\alpha / \beta^{2} c_{1}^{\dagger} c_{2}^{\dagger} c_{3}^{\dagger} c_{4}^{\dagger} c_{5}^{\dagger} c_{6}^{\dagger}$ \\
\hline 001001 & $\alpha \beta c_{5} c_{2}$ & & \\
\hline 000111 & 1 & 001110 & $-c_{5}^{\dagger} c_{6}^{\dagger} c_{3} c_{2}$ \\
\hline 011100 & $c_{1}^{\dagger} c_{6}^{\dagger} c_{4} c_{3}$ & & \\
\hline 101100 & $\alpha \beta^{2} c_{4} c_{3} c_{2} c_{1}$ & 010110 & $\alpha / \beta^{2} c_{1}^{\dagger} c_{2}^{\dagger} c_{5}^{\dagger} c_{6}^{\dagger}$ \\
\hline 001011 & $-\alpha c_{4}^{\dagger} c_{2}$ & 100101 & $-\alpha \beta^{2} c_{6} c_{5} c_{4} c_{1}$ \\
\hline 110010 & $\alpha / \beta^{2} c_{2}^{\dagger} c_{3}^{\dagger} c_{4}^{\dagger} c_{5}^{\dagger}$ & 011001 & $-\alpha c_{1}^{\dagger} c_{5}$ \\
\hline 010101 & $-c_{4}^{\dagger} c_{5}^{\dagger} c_{2} c_{1}$ & & \\
\hline
\end{tabular}
referred to as quantum dimension $d_{i}$. For all bosonic twisted

TABLE I. The first 32 matrix elements of the plaquette operator $Q_{p}$ defined on a hexagon with physical spins $i, j, k, l, m, n$ after ungauging the Hamiltonian given in Ref. [17]. The remaining 32 matrix elements follow by Hermitian conjugation. 


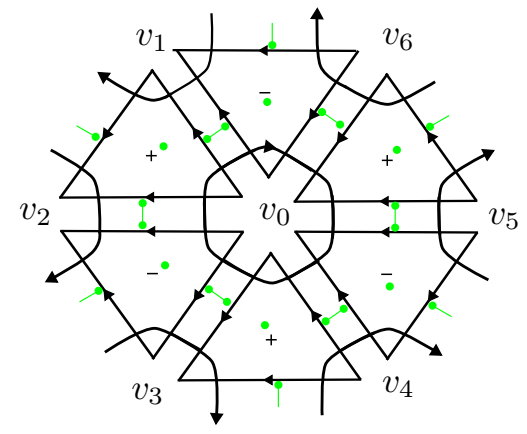

FIG. 12. Hexagonal patch $A_{\text {hex }}$ of a fermionic PEPS with the lattice geometry dual to the physical lattice geometry used in Ref. [18].

quantum double models, an MPO-injective PEPS description can be found. Here, a particular gauge degree of freedom is the choice of the representative $\omega$ of a certain cohomology class which defines the model. A natural gauge is to choose a normalized 3-cocycle, which will yield a wave-function invariant under adding a closed loop, i.e., $d_{i}=1$. Thus, the tensor network gauge suggests a particular Hamiltonian gauge of the corresponding string-net model. The same applies in the fermionic setting.

The Hamiltonian in Ref. [17] is given in a specified gauge $(\beta=1)$. To obtain the gauge compatible with the tensor network description used here, we apply the transformation

$$
c^{\dagger} \mapsto \frac{1}{\sqrt{\beta}} c^{\dagger}, \quad c \mapsto \sqrt{\beta} c,
$$

to ungauge the Hamiltonian, and then choose $\beta=-i$, which yields the gauge with trivial loop weights $d_{i}=1$. The matrix elements in this so-called tensor-network gauge of the plaquette operator, written as

$$
\begin{aligned}
Q_{p}= & \sum_{g_{1}, \ldots, g_{6}} p\left(g_{1}, \ldots, g_{6}\right) \mathcal{F}_{p}\left(g_{1}, \ldots, g_{6}\right) \\
& \times\left|g_{1} \oplus 1, \ldots, g_{6} \oplus 1\right\rangle\left\langle g_{1}, \ldots, g_{6}\right|,
\end{aligned}
$$

where $\oplus$ denotes addition modulo two, are given in Table I. In this gauge, it is easy to check that the tensor network state constructed from the tensors $A_{ \pm}$defined by the triple $\left(\mathbb{Z}_{2}, \omega_{f T C}, s_{f T C}\right)$ is an eigenstate to each plaquette operator. To see this, compute a hexagonal patch of the tensor network $A_{\text {hex }}$ as depicted in Fig. 12 and verify that $Q_{p} A_{\text {hex }}=A_{\text {hex }}$.
[1] B. Zeng, X. Chen, D.-L. Zhou, and X.-G. Wen, Quantum Information Meets Quantum Matter (Springer, Heidelberg, 2015).

[2] R. Orús, Ann. Phys. 349, 117 (2014).

[3] F. Verstraete, V. Murg, and J. I. Cirac, Adv. Phys. 57, 143 (2008).

[4] J. Eisert, Model. Simul. 3, 520 (2013)

[5] J. Eisert, M. Cramer, and M. B. Plenio, Rev. Mod. Phys. 82, 277 (2010).

[6] U. Schollwöck, Ann. Phys. 326, 96 (2011).

[7] N. Schuch, D. Pérez-Garcia, and I. Cirac, Phys. Rev. B 84, 165139 (2011).

[8] X. Chen, Z.-C. Gu, and X.-G. Wen, Phys. Rev. B 83, 035107 (2011)

[9] F. Verstraete and J. I. Cirac, arXiv:cond-mat/0407066.

[10] T. Picot, M. Ziegler, R. Orús, and D. Poilblanc, Phys. Rev. B 93, 060407(R) (2016).

[11] H. N. Phien, J. A. Bengua, H. D. Tuan, P. Corboz, and R. Orús, Phys. Rev. B 92, 035142 (2015).

[12] N. Schuch, I. Cirac, and D. Pérez-Garcia, Ann. Phys. 325, 2153 (2010).

[13] A. Y. Kitaev, Ann. Phys. 303, 2 (2003).

[14] O. Buerschaper, Ann. Phys. 351, 447 (2014).

[15] D. J. Williamson, N. Bultinck, M. Mariën, M. B. Şahinoğlu, J. Haegeman, and F. Verstraete, Phys. Rev. B 94, 205150 (2016).

[16] M. B. Şahinoğlu, D. Williamson, N. Bultinck, M. Mariën, J. Haegeman, N. Schuch, and F. Verstraete, arXiv:1409.2150.
[17] Z.-C. Gu, Z. Wang, and X.-G. Wen, Phys. Rev. B 90, 085140 (2014).

[18] Z.-C. Gu and X.-G. Wen, Phys. Rev. B 90, 115141 (2014).

[19] Z.-C. Gu, Z. Wang, and X.-G. Wen, Phys. Rev. B 91, 125149 (2015).

[20] C.-H. Lin and M. Levin, Phys. Rev. B 89, 195130 (2014).

[21] D. Gaiotto and A. Kapustin, Int. J. Mod. Phys. A 31, 1645044 (2016).

[22] T. Lan, L. Kong, and X.-G. Wen, Phys. Rev. B 94, 155113 (2016).

[23] P. Corboz and G. Vidal, Phys. Rev. B 80, 165129 (2009).

[24] T. Barthel, C. Pineda, and J. Eisert, Phys. Rev. A 80, 042333 (2009).

[25] P. Corboz, G. Evenbly, F. Verstraete, and G. Vidal, Phys. Rev. A 81, 010303(R) (2010).

[26] P. Corboz, Phys. Rev. B 93, 045116 (2016).

[27] C. Pineda, T. Barthel, and J. Eisert, Phys. Rev. A 81, 050303(R) (2010).

[28] P. Corboz, R. Orús, B. Bauer, and G. Vidal, Phys. Rev. B 81, 165104 (2010).

[29] T. B. Wahl, H.-H. Tu, N. Schuch, and J. I. Cirac, Phys. Rev. Lett. 111, 236805 (2013).

[30] J. Dubail and N. Read, Phys. Rev. B 92, 205307 (2015).

[31] C. V. Kraus, N. Schuch, F. Verstraete, and J. I. Cirac, Phys. Rev. A 81, 052338 (2010).

[32] Z.-C. Gu, Phys. Rev. B 88, 115139 (2013).

[33] C. Wille, J. Eisert, and O. Buerschaper (unpublished).

[34] Y. Hu, Y. Wan, and Y.-S. Wu, Phys. Rev. B 87, 125114 (2013). 